\title{
Quelques réflexions à propos de l'exotisme
}

\author{
Marietta Tchoudakova \\ Traducteur : Fanny Mossière
}

\section{CpenEdition \\ Journals}

Édition électronique

URL : http://journals.openedition.org/edl/428

DOI : $10.4000 /$ edl. 428

ISSN : 2296-5084

Éditeur

Université de Lausanne

Édition imprimée

Date de publication : 15 septembre 2009

Pagination : 203-216

ISBN : 978-2-940331-20-8

ISSN : 0014-2026

Référence électronique

Marietta Tchoudakova, "Quelques réflexions à propos de l'exotisme », Études de lettres [En ligne], 2-3

2009, mis en ligne le 15 septembre 2012, consulté le 19 décembre 2020. URL : http://

journals.openedition.org/edl/428 ; DOI : https://doi.org/10.4000/edl.428

(c) Études de lettres 


\section{QUELQUES RÉFLEXIONS À PROPOS DE L'EXOTISME}

Certaines attitudes russes d'aujourd'hui, de la vision esthétisante du guerrier nazi de la dernière guerre à la nostalgie d'un passé soviétique édulcoré, font appel au sentiment d'exotisme afin de réactiver les oppositions entre Nous et les Autres, amis et ennemis, et en même temps de les brouiller en inversant les valeurs. L'analyse du fonctionnement de cet exotisme est complétée dans l'article par le retour vers les années 1920-1930 et la mise en place d'un système dans lequel le récit naturaliste sert de refuge à la liberté créatrice, tandis que le choix de disposer de sa vie à sa manière devient le seul exotisme véritable.

L'exotique, c'est l'autre, l'insolite; ce n'est pas nécessairement l'étranger incarnant une altérité radicale.

Les dictionnaires russes actuels soulignent que ce terme apparaît pour désigner un certain lieu, des pays lointains - en d'autres termes ils lui attribuent une dimension géographique; ils insistent également sur le fait que l'insolite (l'inconnu, la nouveauté) vaut exclusivement pour celui qui perçoit cet exotisme, dans la mesure où il est éloigné du lieu en question, qu'il ne le connaît pas, etc.

Par conséquent, le point depuis lequel on observe a une importance particulière. L'exotisme ne surgit et n'existe en tant que dimension que dans la perception de quelqu'un.

Les bases pour l'attribution de la marque "exotisme" à un objet sont donc les suivantes: 1. la nouveauté pour l'observateur; 2 . l'éloignement de l'observateur: dans le temps (exemples: l'or des Scythes nouvellement découvert ou la tête de Néfertiti) ou dans l'espace.

Cet éloignement est constaté par rapport à l'existence ordinaire: pour percevoir un objet comme exotique, il faut être privé de la possibilité de son observation réitérée et, par conséquent, de la possibilité de s'habituer 
à lui. Un groupe ethniquement différent, une nature inhabituelle cessent d'être exotiques pour celui qui les côtoie quotidiennement. On peut en conclure que la perception d'un objet comme exotique a pour condition d'être superficielle au sens strict du terme: elle doit rester toute extérieure par rapport au phénomène.

Reprenons notre pensée de départ : l'exotique n'est pas l'ennemi. Il est bien plutôt inoffensif. En devenant radicalement étranger et/ou ennemi (c'est-à-dire en entrant dans la situation du sujet percevant qui s'autodétermine), tout mode de vie ou tout individu cesse pour le sujet en question d'être exotique. Tant que la dimension appréciative reste nettement marquée, tant que l'éthique entre en jeu dans le jugement porté sur l'objet, celui-ci ne peut se charger d'exotisme. Il en devient capable, en revanche, si la perception qu'on a de lui est suspendue ou neutralisée sur le plan éthique.

Les jeux autour de ces questions ont commencé depuis longtemps en Russie. L'affirmation de l'auteur du préambule à notre conférence - "Ainsi, l'hitlérisme ne peut pas être présenté comme un exotisme dans la culture russe ${ }^{1}$ - est, hélas, réfutée par la vie russe exotique dès 1973, avec la sortie de la série télévisée Dix-sept instants d'un printemps. L'hitlérisme y est précisément présenté comme exotique; à ce titre, il aura une énorme influence, encore non étudiée à ce jour, sur les mouvements de jeunes nazis et fascisants: l'esthétique des uniformes noirs, des feuilles de chêne argent, des bottes brillantes et de la tenue de parade dissimule le caractère étranger et ennemi de l'objet. Dans de telles représentations du nazisme, l'esthétique engloutit l'éthique: bourreaux et assassins cessent d'être considérés comme "non-nôtres", devenant objets d'admiration, puis d'imitation.

Inversement - les deux mouvements sont probablement liés -, la façon de vivre d'autres peuples, différente, fort éloignée de la manière d'être et de penser de la majorité russe, n'apparaît plus comme exotiquement attirante: elle ne suscite qu'aversion et rejet.

Nos observations nous amènent à constater dans la sémantique de l'exotisme aussi bien la perception esthétique de l'objet que le fait d'en tirer un plaisir, voire de l'admirer.

I. Il s'agit de l'argumentaire rédigé par Leonid Heller pour le colloque Exotismes dans la culture russe (Moscou, juin 2008). Voir l'Avant-propos de ce volume. 
Conformément à ce qui précède (en partant d'ailleurs du Sotsart ${ }^{2}$ ), après Hitler et le nazisme, ce sont Staline et son temps, puis Brejnev et son époque, qui se parent d'exotismes - suite à la fuite de l'information sur la teneur réelle du régime totalitaire.

L'instrument de l'"exotisation", de la transformation du non-exotique en exotique est un puissant levier qui a pour nom "dorure du temps»: elle domine l'opposition nôtre/ non-nôtre.

Cette dernière opposition peut ainsi contribuer à la formation de ce qui rejette l'exotisme, mais tout autant se combiner à sa présence. Et les nôtres, en s'éloignant de plus en plus dans le temps, deviennent également exotiques; ainsi l'époque soviétique, dont les aspects les moins reluisants sont gommés par la propagande de l'actuel gouvernement russe, apparait-elle aujourd'hui d'un exotisme aussi attrayant que l'époque d'Alexandre Nevski.

Je m'apprêtais à rester dans le cadre de la littérature des années trente, mais, en préparant ces notes, j’ai pu me convaincre, comme maintes fois par le passé, que toute étude de l'époque soviétique devait commencer par une incursion, fût-elle brève, dans la vie de l'Empire russe, dans cette période qui précède directement les deux révolutions de 1917, celle de Février et celle d'Octobre.

Dans l'Empire russe orthodoxe, les adeptes d'autres religions et les non-Russes, ont été vus - bien que de manière différente - comme exotiques; les combats qui faisaient rage autour des problèmes de confession, autour de la question juive, autour du "paganisme», activaient les mécanismes de l'exotisation.

Pourtant, le réservoir principal d'exotisme était alors l'Amérique avec ses Indiens (Montigomo Griffe d'épervier ${ }^{3}$ ), les romans de Thomas Mayne Reid et bien d'autres, les îles de l'Océanie et leurs Papous,

2. Courant, dans la peinture, réprimé par la censure officielle, qui détourne à des fins satiriques ou parodiques, les conventions, l'iconographie et les thèmes du réalisme socialiste "étatisé»; ses représentants les plus connus sont Komar et Melamid, auteurs de tableaux "pompiers» à forte dose de surréalisme (NdE).

3. C'est le surnom d'un héros de la nouvelle de Tchékhov intitulée «Les Garçons» («Mal'čiki», 1887), dans laquelle deux garçons jouent aux Indiens et rêvent de fuir leurs maisons pour gagner l'Amérique (NdE). 
l'Afrique (surtout dans les poèmes pour enfants), l'Australie avec ses animaux et ses plantes insolites. Dans la culture russe, les voyageurs ont toujours eu un rôle important à jouer.

A l'autre bout du champ thématique, la prison s'est teintée d'exotisme par le biais des descriptions méticuleuses de Dostö̈evski, Tchekhov et du journaliste Vlas Dorochevitch ${ }^{4}$. Même le principe de l'éloignement y est respecté, en particulier dans les reportages des deux derniers auteurs sur le bagne de Sakhaline.

Après la révolution d'Octobre, l'exotisme littéraire est soumis, d'abord à une pression idéologique, puis à une opération de destruction systématique. L'idée internationaliste de l'union des "prolétaires de tous les pays", provoque une réorientation radicale de l'ordinaire, $\mathrm{du}$ "sien propre» et, partant, de l'exotique. Pendant et après la guerre civile fratricide, pour le Russe, tout autre Russe qui se trouve de l'autre côté de la ligne de front prend l'apparence de l'étranger le plus éloigné, le plus exotique.

A l'inverse, le représentant de toute ethnie appartenant à la Russie désormais soviétique obtient le statut de nôtre et perd sa dimension exotique: premièrement, parce que, peu auparavant, au sein de l'Etat tsariste russe, il faisait figure d'opprimé; deuxièmement, parce qu'il est à présent assimilé au colonisé luttant pour son indépendance et s'apparente, à ce titre, au prolétaire russe.

La mystérieuse et lointaine Grenade n'a dorénavant d'exotique que les sonorités de son nom; elle est maintenant, par essence, proche et «nôtre» 5 .

La littérature du début des années vingt évolue sur un terrain marqué par une série d'oppositions réarrangées: "eux", les étrangers (inostrancy, autres, mauvais) s'opposent "à nôtres», nous qui sommes d'ici, nous qui sommes là aujourd'hui ; étranger (inostranec, habitant hors des frontières du pays) s'oppose à Russe, citoyen de la Russie révolutionnaire; enfin,

4. Il est fait ici référence aux Souvenirs de la maison des morts (1862) de Dostö̈evski (dernière édition française: Carnets de la maison morte, Arles, Actes sud, 1999), Ostrov Sahalin (1895) de Tchékhov (L'Ile de Sakhaline, Grenoble, Cent pages, 1995) et de Vlas Dorochévitch, Sahalin (1901; traduction française: L'Ile des morts. Les forçats de Sakhaline, Paris, Ambert, s. d.) (NdE).

5. "Grenade» (1926) : poème de Mikhaïl Svetlov, exemple classique de la tendance romantique et internationaliste de la poésie soviétique (NdE). 
étranger ( $\check{c} u z ̌ o j$, bourgeois, capitaliste, exploiteur) se confond avec Russe d'avant, Russe garde-blanc, officier, porteur d'épaulettes dorées, émigré.

Le fait d'être propre, soigné, de porter un beau costume est, du même coup, dévalorisé et associé à l'étranger, au "mauvais».

La nouvelle de Vsevolod Ivanov «Dite» ("L'Enfant», 1922) explique parfaitement cette nouvelle manière de voir. Dans les steppes sauvages de la Mongolie, une bande de partisans "rouges" attaque un convoi «blanc» et adopte un nourrisson survivant; on lui trouve une nourrice kirghize, dont on tue le bébé, afin qu'elle puisse consacrer tout son lait à sauver l'enfant "chrétien". Ce dernier est ainsi opposé à la fois à son père russe contre-révolutionnaire, qui mérite d'être abattu, et à un bébé "ethniquement autre» ("une racaille basanée»), kirghize, que l'on doit supprimer pour sauver le "nôtre ${ }^{6}$. La littérature soviétique sera friande de ces schémas narratifs.

Dans le récit de Rouvim Fraerman, Nikitchen (1933), qui raconte la vie des Evenks, peuple du Grand-Nord, on trouve le dialogue suivant:

... La petite fille demanda:

- Qui sont ces visiteurs venus avec vous à dos de rennes?

- Ce sont des Rouges, répondit Olechek.

- Ils sont blancs, déclara Nikitchen avec obstination, se souvenant des pantalons de grosse toile des Russes.

- Ils se nomment eux-mêmes Rouges, ce qui, chez eux, veut dire hommes blancs. Ils ont chassé des mines les soldats et les maîtres, et maintenant, ils se battent contre les Japonais 7 .

On le voit, une réorganisation des oppositions ethniques traditionnelles est à l'œuvre. Ce qui est russe (en tant que prérévolutionnaire, douteux, tendant au signe «moins») se retrouve, au pôle de l'altérité, face à ce qui n'est pas russe (international, actuel) mais qui a de la valeur et, en fin de compte, est plus «nôtre» que nous-mêmes:

6. Précisons que, dans la nouvelle, une distance est maintenue entre le monde quasi sauvage de la lutte pour la survie biologique, à peine touché par les bouleversements idéologiques, et les positions du narrateur comme du lecteur implicite ( $\mathrm{NdE})$.

7. «Devočka sprosila: - Kto oni, èti gosti, prišedšie s vami na olenjah? - Krasnye, - otvetil Olesek. - Oni belye, - uprjamo skazala Nikičen, vspomniv parusinovye štany russkih. - Oni nazyvajut sebja krasnye, čto značit po-ihnemu belye ljudi. Oni prognali s priiskov soldat i hozjaev i teper' vojujut s japonsami». R. Fraerman, Nikičen. Vas'ka Giljak, p. 15. 
Dans la blanche salle du palais

L'accent non russe du président paraît étrangement autre

Oh ces gens durs comme la pierre

On les vénérera dans les siècles et les siècles... ${ }^{8}$

Il se peut que l'intérêt pour la littérature traduite - manifeste dès avant la révolution - s'amplifie, allant jusqu'au camouflage de livres d'aventure russes en traductions. Dans ce contexte, la littérature traduite est tout à la fois attirante et repoussante.

Dans son article «Sur la littérature d'aventures et sur les nouveaux écrivains", le critique Abram Lejnev écrit:

La description du quotidien engendre déjà une réaction contre ellemême. Cette réaction, c'est la littérature d'aventure. Elle ne surmonte pas la description du quotidien, comme le fait le roman qui est né de ce quotidien et le dépasse. Elle le repousse, elle ne le prend pas en considération. Elle ne date pas d'aujourd'hui, mais, récemment encore, elle demeurait tapie à l'arrière-plan, avançait par la bande, était considérée comme une parente pauvre de la famille littéraire. Aujourd'hui, en revanche, elle est universellement reconnue. Notre littérature d'aventure est restée à la traîne de la littérature étrangère. Les romans et les récits d'aventure russes sont plus ennuyeux que ceux des autres pays, et sont faits de manière plus grossière. Le lecteur russe lit plus volontiers Burroughs et Benoit que I. Gontcharov et A. Tolstoï. Cependant, le fait même que Tolstoï ait écrit des romans d'aventure a permis à ce genre littéraire de remporter une immense victoire: il a cessé d'être une littérature inférieure, une «littérature de boulevard». Cette victoire est celle du goût pour l'exotique?

Dans les années vingt, deux œuvres marquantes traitent de manière opposée le rapport à cet Orient qui constitue traditionnellement un

8. «I stranno čužd v dvorcovom zale belom / Nerusskij predsedatelja akcent / O èti ljudi, tverdye kak kamen’ / ... / Ih budut čtit' vekami i vekami.» V. Stenič, «Narkomvoen otryvisto čekanit...».

9. A. Ležnev, "Russkaja literatura v istekšem godu», p. 65. Edgar Rice Burroughs, inventeur de Tarzan et d'aventures sur Mars et sur Vénus, et Pierre Benoit, auteur de L'Atlantide, comptent parmi les écrivains les plus édités et les plus lus en Russie à l'époque de la NEP (NdE). 
réservoir d'exotisme pour la culture russe: une des lignes importantes de L'Appartement de Zö̈ka ${ }^{10}$ de Boulgakov (qui prend la suite de sa nouvelle de 1922, "Une Histoire de Chinois»), menée dans une tonalité quasi burlesque, représente en réalité une vive polémique avec le récit de Vsevolod Ivanov Le Train blindé $n^{0} 14-69^{11}$, mettant en scène un Chinois héroïque qui se sacrifie pour la révolution mondiale.

A la fin des années vingt et au début des années trente, la critique de la RAPP (Association russe des écrivains prolétariens) veille scrupuleusement à ce que les républiques périphériques ne soient pas vues comme exotiques. Un critique de La Sentinelle littéraire, la revue "prolétarienne» la plus orthodoxe, reproche à Andreï Biély, dans son livre Le Vent du Caucase ${ }^{12}$, de "réduire la culture nationale à l'exotisme et à l'ethnographie " ${ }^{13}$. La même critique est alors adressée au Voyage en Arménie de Mandelstam (1933) ${ }^{14}$.

Simultanément, pourtant, on observe un phénomène qui prend le contre-pied des exigences de la "ligne générale»: le champ littéraire cherche toujours à se nourrir d'exotisme. Ainsi Dmitri Mirski, venu en Union soviétique depuis l'Angleterre, fait-il activement connaître, jusqu'à son assassinat par le pouvoir soviétique, la poésie et la prose de Kipling au lecteur russe; il contribue ainsi à la diffusion du goût de l'exotisme.

La pression de la censure n'empêche pas un Vitali Bianki d'évoquer en détail la conception de la nature d'une fille Khakasse dans «Emuranki» («Les Gerboises», 1936). De la même manière, le récit de Rouvim Fraerman déjà mentionné, Nikitchen, malgré une part d'idéologisation, retrace la vie quotidienne et la psychologie des Evenks (alors appelés Toungouses) comme un monde exotique au sens propre, c'està-dire inhabituel et captivant. Fraerman écrit son chef-d'œuvre, le récit pour enfants Dingo, chien sauvage, ou Récit d'un premier amour ${ }^{15}$, dans

Io. M. Bulgakov, Zojkina kvartira (1925). Cf. M. Boulgakov, L'Appartement de Zoika.

II. V. Ivanov, Bronepoezd 14-69. Cf. Le Train blindé n¹4-69.

I2. A. Belyj, Veter s Kavkaza. Vpečatlenija.

I3. S. Amaglobeli, "Kuda duet veter? ("Dans quelle direction souffle donc ce vent?»), p. 24.

I4. O. Mandelstam, Voyage en Arménie.

I5. R. Fraerman, Dikaja sobaka Dingo ili povest' o pervoj ljubvi. Cf. Tania ou le Premier amour. 
lequel la vigilance des organes de contrôle est déjouée, précisément, grâce au pittoresque de l'Extrême-Orient, c'est-à-dire à l'exotisme de la nature, des rennes, ainsi qu'à celui des ethnies minoritaires (le garçon Nénèts Filka et son père, proposant «à Tania sa petite pipe pour qu'elle fume, car il était un homme bon»), dont le retour sur le devant de la scène est favorisé par la fin de l'internationalisme et la disparition des espoirs d'une révolution mondiale. Au lieu du «récit d'écolier» soviétique attendu, on a ici une analyse très fine des sentiments humains, pratiquement libre de tout soviétisme.

D'autre part, un nouvel aspect de l'américanisme s'affiche, et sa force d'attraction est décuplée. Déjà, avant la révolution, Alexandre Blok voyait la Russie comme une nouvelle Amérique:

... Ainsi, sur la steppe verte j’ai vu s'allumer

L'étoile de la Nouvelle Amérique ${ }^{16}$.

Dans l'avant-propos de son poème Châtiment (Vozmezdie, 1911-1919), il insiste:

...La Russie va vers la nouvelle Amérique, la nouvelle, et non l'ancienne... ${ }^{17}$.

Le pouvoir soviétique $\mathrm{a}$, en quelque sorte, repris l'idée du progrès technologique effréné du Nouveau Monde; on comparera, à cet égard, le rapport de Maïakovski, dans ses notes de voyage, respectivement à l'Europe et à l'Amérique (1925) ${ }^{18}$. De même, plus tard, dans le reportage d'Ilia Ilf et Evgueni Petrov L'Amérique sans étages (Odnoètažnaja Amerika, 1937) ${ }^{19}$. Et jusque dans les Eufs fatidiques (Rokovye jajca, 1925) ${ }^{20}$ de Mikhaïl Boulgakov, certains critiques bienveillants décèlent le rythme du Nouveau Monde.

I6. "...To nad step'ju pustoj zagorelas'/ Mne Ameriki novoj zvezda». A. Blok, «Novaja Amerika», p. 396-398.

17. "...Rossija - v novuju Ameriku, v novuju, a ne staruju Ameriku». A. Blok, Préface à "Vozmezdie», t. 3, p. 479.

I8. V. Majakovskij, Moe otkrytie Ameriki. Cf. V. Maïakovski, Du monde j’ai fait le tour.

19. I. Ilf et E. Petrov, Odnoètažnaja Amerika. Cf. I. Ilf et E. Petrov, Amérique sans étages; id., L'Amérique: roman-reportage.

20. M. Bulgakov, Rokovye jajca (interdit ensuite, le récit ne sera réédité en URSS qu’à partir de 1989). Cf. M. Boulgakov, Les Eufs fatidiques. Diablerie et autres récits. 
Vers le début des années trente, la vie sociale des individus est déjà entièrement réglementée en tant qu’objet de représentation littéraire. La représentation des choses, des objets reste cependant assez «libre» de toute règle; c'est elle que choisissent certains auteurs d'očerk (reportage "productiviste»), poursuivant leur travail dans une impasse, car ce genre n'est pas destiné à faire l'inventaire des objets en dehors des travailleurs, des êtres humains.

Dans une plus grande mesure encore, la représentation des animaux et des paysages demeure également libre. Ce nouveau type de récits fait appel à un auteur-observateur - géographe, naturaliste ou chasseur. En réduisant au minimum le dialogue de l'individu avec ses semblables (les échanges se limitent aux nécessités de la chasse et de la pêche), le sujet de ces récits contourne aussi les règles en vigueur: on n'a pas encore eu le temps de réglementer les relations de l'homme avec les arbres ou les lièvres.

Les portes de la littérature s'ouvrent alors, de manière inattendue, sur les «zones inhabitées", sur la flore et la faune du désert et de la taïga. Devenant le lieu de l'action, les terres éloignées du centre du pays dressent une barrière invisible qui les protège de la censure soviétique déjà passablement perfectionnée et, par la même occasion, des clichés narratifs et idéologiques obligés. Les régions périphériques, peu peuplées, offrent aux auteurs une certaine liberté de manœuvre dans la construction de l'intrigue et la thématique, bien que la critique continue de tirer périodiquement à boulets rouges sur la philologie et la littérature, dans la guerre qu'elle mène contre le "formalisme» (l'ambiguïté même du terme ouvre de larges perspectives; l'un de ces «bombardements» affectera douloureusement la littérature en 1932-1933).

L'intérêt pour les livres de Vladimir Arseniev ${ }^{21}$, datant du début des années vingt, revient rapidement. Mais l'ensemble des circonstances fait que, dans le domaine de l'art, le vecteur social se trouve dirigé, au début des années trente, vers les formes courtes. L'évolution littéraire va chercher à donner une nouvelle forme à la nouvelle.

2I. V. Arsen'ev, Dersu Uzala. Iz vospominanij o putešestvii po Ussurijskomu kraju 1907g. Cf. V. Arseniev, Dersou Ouzala. La Tä̈ga de l'Oussouri. 
Mikhaïl Prichvine fixe tout ce processus de manière laconique et éloquente, dans son discours au plénum du Comité d'organisation du futur congrès des écrivains, à l'automne 1932:

Voilà qu'on m'adresse un mot de la rédaction: vous ne nous écririez pas un petit récit sur des chiens ou quelque chose de ce genre? On dirait que quelque chose revient, on sent un léger parfum de liberté (Rires) $^{22}$.

La dernière phrase parait comique aux auditeurs-littérateurs, mais elle ne peut leur paraître absurde. La liberté d'écrire sur les chiens est, dans une certaine mesure, une conquête; le diktat du thème relâche légèrement son étreinte.

Finalement, le récit sur la nature et les animaux, traités comme exotiques, devient l'unique possibilité de philosopher sur des thèmes liés à l'existence humaine.

L'exemple le plus caractéristique en est le récit de Prichvine, Ginseng (1933) ${ }^{23}$, qui évoque la vie dans les régions maritimes subtropicales, au cœur d'une nature "d'une ineffable beauté», exotique pour l'Européen, en étroite proximité avec de magnifiques animaux et aux côtés des Chinois, inévitablement exotiques pour les Russes.

Les "récits de chasse" - c'est-à-dire, pour de nombreux lecteurs, des récits sur des thèmes exotiques - doivent être compris comme une réaction non seulement à l'urbanisation, mais aussi, et surtout, à l'idéologisation de toute la vie soviétique, qui se renforce de plus en plus dans les villes. L'intérêt pour le récit de chasse grandit fortement à la fois chez les écrivains et chez les lecteurs pour une autre raison encore: la "couleur locale" de la chasse permet de charger de détails sanglants l'intrigue, basée soit sur la mort de l'animal, soit sur celle de l'homme. C'est ainsi que ressurgissent dans la littérature - comme dans les récits de Bianki, «La Gélinotte» (1934), «Djoulbars» (1936) ${ }^{24}$ - les thèmes refoulés et interdits de la réalité sanglante du temps.

22. M. Prišvin, doklad, in Sovetskaja literatura na novom ètape. Stenogramma I-go plenuma Orgkomiteta Sojuza sovetskih pisatelej, p. 116.

23. M. Prišvin, Žen' Šen'-koren' žizni. Cf. M. Prichvine, Ginseng. La racine de vie et autres récits d'un chasseur russe.

24. Cf. V. Bianki, «Emuranki», «Rjabčik», «Džul’bars». 
En 1937, Prichvine lit en traduction allemande «un livre remarquable d'un Indien, Le Hibou Gris - Grey Owl» (c'est ainsi qu'il l'écrit dans son journal) et conclut:

[...] je pense que je suis bien meilleur que lui comme écrivain, mais qu'il est bien meilleur que moi comme homme ${ }^{25}$.

Sa décision de réécrire ce livre en russe est un fait intéressant de la génétique ou de la téléologie littéraire, et marque probablement l'apogée de l'intérêt pour l'exotisme d'un pays certes lointain, mais nullement éloigné dans le temps, et pour la vie exotique d'un contemporain du lecteur soviétique.

Paru en 1938 dans la revue Molodaja gvardija (La jeune Garde) et en édition séparée en $1939^{26}$, le livre de Grey Owl, réécrit par Prichvine, est, naturellement, un événement important de la vie littéraire russe; son succès démontre qu'un monde animal et un monde humain, dans lesquels les relations avec la nature se bâtissent librement, fascinent à la fois les lecteurs et les écrivains.

Cette recherche littéraire de relations libres, non-réglementées entre l'homme et l'animal est très éloignée de la «littérature du dressage» que certains exégètes d'une "utopie de l'animalité» classent aujourd'hui dans une "catégorie littéraire distincte» (en y incluant Prichvine), comme une écriture qui remplit «des fonctions idéologiques particulières, parfois très importantes ${ }^{27}$.

A un niveau superficiel, l'exotisme reste toujours la nature vierge et sauvage (les héros tentent de la préserver de la destruction); toutefois, à un niveau plus profond - et avec une puissance très grande -, vers la fin des années trente, ce n'est plus la nature qui apparaît comme exotique, mais la vie privée et la liberté d'action individuelle. On ne peut alors satisfaire à cette exigence du lecteur que dans un cercle très restreint de possibilités. Le récit pour enfants en est une; par exemple, le héros

25. "...Tak sčitaju, čto ja bol'še ego mnogo kak pisatel', no on bol'še menja kak čelovek». M. Prišvin, Dnevniki. 1937, p. 311.

26. Seraja Sova. [Vèsa Konnezin], pereskaz Mihaila Prišvina. Cf. en allemand Wäscha-kwonnesin (Grau-Eule), Der heilige Baum. Erlebnisse aus dem kanadischen Norden; en français G. Owl, Ambassadeur des bêtes. Il s'agit de Grey Owl ou Wa-shaquon-asin, pseud. de Archibald Belaney, écrivain canadien qui adopta le mode de vie indien (NdE).

27. L. Geller (Heller), «Predislovie. Životnye v reprezentacijah», p. 12. 
de Timour et sa bande (1939) d'Arkadi Gaïdar se distingue des personnages «adultes» de ce même livre et de bien d'autres, tant par le fait qu'il agit à sa guise que par sa volonté d'assumer la responsabilité de ses actes individuels.

Une autre possibilité est la réécriture d'un livre consacré à la manière de vivre des gens d'une autre civilisation. Le Grey Owl de Prichvine est l'apothéose de la liberté qu'a l'être humain de disposer de sa propre vie - ce qui était perdu depuis longtemps et, semblait-il, irrévocablement, pour le lecteur et l'écrivain russes dans leur pays.

\author{
Marietta Tchoudakova \\ Moscou \\ Traduction Fanny Mossière
}




\section{BIBLIOGRAPHIE}

Amaglobeli, S., «Kuda duet veter?», Na literaturnom postu, 16 (1931). Arseniev, V., Dersou Ouzala. La Taïga de l'Oussouri, traduction de Pierre Wolkonsky, Paris, Pygmalion, 1997.

Belyj, A., Veter s Kavkaza. Vpečatlenija, Moskva, Federacija, [1928].

Bianki, V., «Emuranki», «Rjabčik», «Džul'bars», in Sobranie socinenij, t. 2, Leningrad, Detskaja literatura, 1973.

BцoK, A., "Novaja Amerika», in Sobranie sočinenij v 8 tt., p. 396-398.

—, Préface à "Vozmezdie», t. 3, p. 479.

Boulgakov, M., Les Eufs fatidiques. Diablerie et autres récits, Paris, Librairie française, 1988.

—, L'Appartement de Zö̈ka, in Euvres, vol. 2, Paris, Gallimard, Bibl. de la Pléïade, 2004.

Fraerman, R., Nikičen. Vas'ka Giljak, Moskva, Molodaja gvardija, 1933.

—, Dikaja sobaka Dingo ili povest'o pervoj ljubvi, Moskva, Sov. pisatel', 1939; traduction française de Fabienne Finifter: Tania ou le Premier amour, Paris, éd. de la Farandole, 1974.

Geller (Heller), L., "Predislovie. Životnye v reprezentacijah», in Utopija zverinosti. Reprezentacii životnyh v russkoj kul'ture, ed. Geller (Heller), Leonid, Drohobycz, Kolo, 2007.

Ilf, I. et Petrov, E., Amérique sans étages, traduction de Ludmila Savitzky, Paris, éd. du Pavois, 1946.

-, L’Amérique: roman-reportage, traduction d'Alain Préchac, Paris, Parangon, 2002.

Ivanov, V., Le Train blindé no 14-69, traduction de Sidersky, Paris, Gallimard, 1927.

Ležnev, A., "Russkaja literatura v istekšem godu», (s. 1.), Pisatel’ $i$ revoljucija, 1 (1926).

Majakovskij, V., Moe otkrytie Ameriki, Moskva, GIZ, 1926; traduction française: Du monde j'ai fait le tour, Paris, La Quinzaine littéraire, 1998. 
Mandelstam, O., Voyage en Arménie, traduction d'André du Buchet, Paris, Mercure de France, 2005.

Prichvine, M., Ginseng. La Racine de vie et autres récits d'un chasseur russe, traduction de G. Welter, Paris, Payot, 1946.

PRIŠvin, M., doklad, in Sovetskaja literatura na novom ètape. Stenogramma I-go plenuma Orgkomiteta Sojuza sovetskih pisatelej, Moskva, Sovetskaja literatura, 1933.

—, Dnevniki. 1937, Oktjabr', 1994, 11, 1995, 9.

Seraja Sova. [Vèsa Konnezin], pereskaz Mihaila Prišvina, Moskva/ Leningrad, Detgiz, 1939.

Stenič, V., «Narkomvoen otryvisto čekanit...», in Sopo. Pervyj sbornik stihov, [Moscou], 1921.

Wäscha-KWonnesin (Grau-Eule), Der heilige Baum. Erlebnisse aus dem kanadischen Norden, Stuttgart, Kosmos, 1937. 